\title{
La interpretación del derecho en Colombia a la luz de los postulados neoconstitucionalistas
}

\section{The interpretation of the law in Colombia in the light of the neoconstitutional postulates}

\author{
DOI: https://doi.org/10.17981/juridcuc.17.1.2021.21
}

Fecha de reflexión: 2020/06/13 Fecha de Aceptación: 2021/02/27

\author{
Yamal Elías Leal Esper \\ Universidad Libre de Colombia. Cúcuta (Colombia) \\ yamale.leale@unilibre.edu.co
}

Para citar este artículo:

Leal, Y.(2021). La interpretación del derecho en colombia a la luz de los postulados neoconstitucionalistas. Jurídicas CUC, 17(1), 613-628. DOI: http://dx.doi.org/10.17981/juridcuc.17.1.2021.21

\section{Resumen}

El Estado Constitucional, durante varios años ha reclamado una nueva teoría del derecho, que se separa del tradicional esquema del positivismo teórico, basado en la subsunción como método de interpretación para dar solución de los diferentes problemas jurídicos que afectan a la sociedad; dicha teoría es denominada "Neoconstitucionalismo", concepto que explica un fenómeno reciente dentro del Estado Constitucional contemporáneo que presenta una nueva actitud interpretativa del derecho fundada en las ideas de supremacía del texto constitucional, su fuerza vinculante y garantía jurisdiccional. En este orden de ideas, el objetivo general de este artículo de reflexión, es analizar si en Colombia se interpreta el derecho de acuerdo a los postulados neoconstitucionalistas y que influencia han ejercido en la práctica del derecho. Para cumplir este objetivo, se utilizó una metodología cualitativa con enfoque documental, llevando a cabo una revisión bibliográfica donde diversos tratadistas contemporáneos han estudiado la influencia neoconstitucional en el sistema jurídico colombiano, a partir del papel que desempeñan los jueces al hacer el ejercicio de interpretar el derecho en el momento de resolver los llamados casos difíciles. Finalmente, la respuesta al objetivo general se da en tres grandes subtítulos: el primero de ellos es un abordaje teórico sobre el término neoconstitucionalismo como teoría jurídica; el segundo es la presentación de sus más importantes postulados, y el tercero permite apreciar si en Colombia la interpretación del derecho se realiza a la luz de los principios neoconstitucionalistas, para luego hacer una discusión al tema, y las conclusiones de la investigación.

Palabras clave: Aplicación directa; carácter vinculante; fuerza normativa; garantía judicial interpretación; neoconstitucionalismo

\begin{abstract}
The constitutional state, for several years has called for a new theory of law, which is separated from the traditional scheme of theoretical positivism, based on subsuntion as a method of interpretation to solve the different legal problems affecting society; This theory is called "Neoconstitutionalism", a concept that explains a recent phenomenon within the contemporary Constitutional State that presents a new interpretative attitude of law based on the ideas of supremacy of the constitutional text, its binding strength and judicial guarantee. In this order of ideas, the general objective of this article of reflection is to analyze whether in Colombia the right to the light of neoconstitutional postulates is interpreted and influenced in the practice of law. To achieve this objective, a qualitative methodology with a documentary approach was used, carrying out a bibliographic review where various contemporary traffickers have studied the neoconstitutional influence on the Colombian legal system, based on the role that judges play in exercising the exercise of interpreting the right at the time of resolving so-called difficult cases. Finally, the answer to the general objective is given in three major subtitles: the first is a theoretical approach to the term neoconstitutionalism as a legal theory; the second is the presentation of its most important postulates, and the third makes it possible to appreciate whether in Colombia the interpretation of the law is carried out in the light of neoconstitutional principles, and then to make a discussion on the subject, and the conclusions of the investigation.
\end{abstract}

Keywords: Direct application; binding nature; regulatory force; judicial guarantee andnterpretation; neoconstitutionalism 


\section{INTRODUCCIÓN}

El tema de la influencia neoconstitucionalista en el ordenamiento jurídico colombiano no es precisamente una novedad; pero, si un argumento necesario y trascendental, para ser puesto en discusión cuando se trata de afirmar su influjo en direccionar las decisiones en el derecho moderno. Siendo esta una razón por la cual Carbonell y García (2010), han afirmado que el neoconstitucionalismo:

[...] ha enmarcado la consolidación y expansión de la democracia constitucional, ha reforzado la vigencia del argumento de los principios en el derecho y ha incidido dramáticamente en la configuración de la concepción contemporánea de los derechos fundamentales, con hondas repercusiones en la práctica de su aplicación (párr. $1)$.

Esa influencia constitucional hace que los jueces constitucionales tengan que aprender a realizar su función sobre la base de técnicas interpretativas nuevas donde los principios como pro personae, o los derechos fundamentales, cobran especial importancia volviendo esta tarea más compleja utilizando para ello las técnicas de la ponderación o la proporcionalidad.

En ese orden de ideas los jueces enfrentan dificultades al tener que trabajar con valores plasmados en el texto constitucional, pues al aplicarlos requieren de una tarea de hermenéutica que sea capaz de colocar estos valores a los casos concretos de forma justificada y razonable, razones estas que evidencian la importancia de conocer los postulados del neoconstitucionalismo para la interpretación del derecho como por ejemplo que el texto constitucional no puede ser modificado sino a través de un proceso extenso en virtud de la rigidez constitucional que pregona el neoconstitucionalismo o el carácter vinculante de las normas del texto constitucional para los operadores jurídicos y/o funcionarios del poder público para el caso Colombiano. 


\section{Discusión}

\section{Conceptualización del neoconstitucionalismo como teoría jurídica}

A pesar del abundante material que con respecto del tema se ha visto en los últimos años, o tal vez justamente por dicha profusión, resulta difícil encontrar una definición concisa del neoconstitucionalismo, o del movimiento neoconstitucional. Cada autor parece centrar su enfoque sobre diversos aspectos, añadiendo así nuevas características a un concepto que pareciese demasiado amplio y difuso (García, 2009).

Sin embargo, el análisis de los diversos planteamientos permite estudiar cómo se establecen una serie de elementos comunes que vienen, a la larga, a constituir un consenso sobre las características principales que configuran la doctrina neoconstitucional. De esta forma, Gil (2011) llega a una definición simple, pero ilustradora: el neoconstitucionalismo es, en esencia, "una nueva visión del Estado de Derecho" (p. 43), propia de la segunda mitad del siglo XX, y constituida a partir del principio de la "supremacía de la constitución sobre todas las demás normas jurídicas" (Vásquez y Barrios, 2018, p. 157). Es decir, que las políticas constitucionales vienen a convertirse ahora en los principios activos que dirigen todo el accionar legislativo de una nación.

Esto, en sí mismo, podría parecer a primera vista poco novedoso. A fin de cuentas, la idea de la constitución a modo de norma de normas hace parte del constitucionalismo clásico. Sin embargo, el punto se halla evidente al comparar los textos constitucionales mismos. Las constituciones antiguas se caracterizaban por la brevedad de su contenido y la puntualidad de su articulado, por lo cual, la actividad legislativa posterior era la que venía realmente a regir la acción estatal. Comparadas con los manuscritos legislativos extensos de las diversas áreas, las constituciones clásicas tenían algo muy mínimo que aportar, así que eran estos códices los que orientaban en la práctica, el accionar judicial y administrativo de los Estados. Por el contrario, las cartas legislativas modernas, mucho más extensas y detalladas que 
sus predecesoras, han tomado un papel muy activo en la vida jurídica contemporánea, tanto a nivel individual; dotando al ciudadano de mayores herramientas para su participación y protección de sus derechos, como a nivel gubernamental; estableciendo principios rectores que deben guiar todos los actos gubernamentales.

Siguiendo esta idea, Comanducci (2002) considera que los múltiples postulados neoconstitucionalistas, que han tenido lugar en los últimos años; buscan explicar la naturaleza del derecho constitucional moderno que en las últimas décadas se ha separado ampliamente del derecho constitucional clásico. Así, son estos cambios entre la doctrina clásica y la contemporánea los que vienen a definir la esencia de la doctrina neoconstitucional, y por lo tanto, deben ser el punto de partida para el estudio de la misma.

\section{Postulados neoconstitucionalistas} para la interpretación del derecho

Con el fin de identificar los postulados neoconstitucionalista se ha profundizado en las diferencias entre las constituciones clásicas y las contemporáneas. Siguiendo está mecánica, Prieto (2003) ha procurado resumir el neoconstitucionalismo, estableciendo un conjunto de 6 características fundamentales que lo especifican:

(i) La fuerza vinculante: no se pretende que las constituciones sean códigos morales, o conjuntos de simples preceptos, sino normas vinculantes. (ii) Normatividad superior: no son sólo normas, sino normas de normas, y toda la validez de las demás medidas quedan condicionadas a la constitución. (iii) Aplicación directa: no se necesita de ningún otro acto jurídico para que la constitución ejerza su fuerza vinculante. (iv) Garantía Judicial: la constitución se convierte en una herramienta de control, tanto anterior, como posterior del accionar judicial, en manos de tribunales especializados (la jurisdicción constitucional) y de jueces ordinarios. (v) Contenido normativo: como ya se mencionó, las constituciones contemporáneas tienen un extenso contenido normativo, que abarca principios de la actividad estatal, derechos y deberes de los ciudadanos, y directrices de la actividad legislativa, entre otras cosas. (vi) Rigidez constitucional: se requiere 
de un proceso extenso y metódico, usualmente complejo, para cambiar el contenido constitucional.

Si bien su contenido es debatible, especialmente en lo que respecta a su "novedad", estos seis preceptos son un excelente ilustrador de las características de las constituciones contemporáneas que han generado el neoconstitucionalismo, ya que; muestran con claridad la mencionada supremacía de la constitución sobre la cual dicha corriente está constituida. Sin embargo, para Carbonell (2006), al estudiar el neoconstitucionalismo, y a pesar de su importancia, no basta con la simple revisión del texto constitucional; sino que también, se hace necesario aprender dos elementos que se derivan de este y tienen a su vez un carácter propio: la jurisprudencia y los desarrollos teóricos, la primera en manos de los jueces y la segunda de los académicos del derecho.

Ahora bien, sobre la primera, el nuevo constitucionalismo ha tenido una influencia clara en el accionar de los jueces ordinarios, quienes se ven en obligación de establecer frente a criterios y herramientas constitucionales dentro de su actividad cotidiana (Prieto, 2001); la garantía judicial mencionada de las constituciones contemporáneas. Es en los tribunales constitucionales, donde, se ha visto el mayor dominio, porque los jueces han necesitado que aprender a realizar su función bajo nuevos parámetros, de carácter cada vez más complejo. (Barak, 2006).

No obstante, en muchos casos, las constituciones contemporáneas llevaron a la creación de jurisdicciones reglamentarias diferenciadas y especializadas; como la Corte Constitucional en el caso colombiano a partir de la Constitución Política (CP, 1991).

Por su parte, en el caso de los desarrollos teóricos, estos se alimentan a su vez de los dos aspectos mencionados: texto constitucional y jurisprudencia, pero no se limitan a ellos. Los postulados teóricos del neoconstitucionalismo no buscan limitarse al estudio de lo ya existente, sino que vienen aderezados de un fuerte carácter propositivo, que busca desarrollar y complementar el contenido revisado (Carbonell, 2018).

Es decir, se presenta un proceso de retroalimentación entre "la teoría y la práctica [del derecho]" (León, 2015, p. 20). Sí bien este papel de la teoría como elemento forjador del derecho no es, en sí mismo, para nada algo novedoso, la forma en como los nuevos jueces constitucionales 
recurren a la hora de dictar sentencia, cada vez con menos temor, a contenidos y herramientas elaboradas por la teoría, sí puede ser considerada en sí misma a modo de novedad.

Es importante resaltar que los diferentes ordenamientos jurídicos vienen sufriendo una serie de cambios al punto de que se hable de la "constitucionalización del ordenamiento jurídico", fenómeno que ha sido definido por Guastini (citado por Marín y Zuluaga, 2013) como:

[...] un proceso de transformación de un ordenamiento, al término del cual, el ordenamiento en cuestión resulta totalmente impregnado por las normas constitucionales. Un ordenamiento jurídico constitucionalizado se caracteriza por una Constitución extremadamente invasora, entrometida, capaz de condicionar tanto la legislación como la jurisprudencia y el estilo doctrinal, la acción de los actores políticos así como las relaciones sociales (pp. 146-147).

Sobre la constitucionalización del ordenamiento jurídico, se debe tener en cuenta que para establecer si un ordenamiento jurídico se ha constitucionalizado se deben dar las siguientes condiciones: una "constitución rígida, la garantía jurisdiccional [...], la fuerza vinculante, la aplicación directa de la norma constitucional, la interpretación conforme de las leyes, y la influencia de la constitución sobre las relaciones políticas" (Carbonell, citado en Mattos, 2001, p. 53).

Sobre la Constitución rígida, la constitucionalización será más acentuada en aquellos ordenamientos en los que existan principios que no pueden ser modificados ni siquiera mediante los procedimientos de reforma constitucional previstos en la misma constitución, (A manera de ejemplo en el caso colombiano se podrá reformar la constitución vía congreso de la república). El propio Guastini (2007) expreso "sobre este rasgo que la constitucionalización será más acentuada en aquellos ordenamientos en los que existan principios (tanto expresamente formulados como implícitos) que no puedan ser modificados en modo alguno: ni siquiera mediante el procedimiento de revisión constitucional" (p. 155).

Respecto de la fuerza vinculante de la Constitución, se concreta en el hecho de que las normas constitucionales son aplicables y constriñen 
a sus destinatarios, y sus alcances interpretativos dependerán de las decisiones que haya tomado la jurisdicción constitucional, que hacen que dichas normas se apliquen directamente.

\section{La interpretación del derecho a la luz del neoconstitucionalismo}

Ahora bien, se tiene por cierto que la interpretación del derecho se puede entender como las actividades posteriores a la promulgación de las normas que permiten analizar su validez, estimar un principio general de derecho, o motivar una sentencia (Saavedra, 1994). Conforme a la luz del neoconstitucionalismo la interpretación del derecho necesariamente ha cambiado su orientación, teniendo en cuenta, la importancia vital que se da a la Constitución, no sólo como referente normativo, sino a modo de norma de carácter supra jurídico, cuyo contenido, se constituye en un referido para otras reglas, además de principios y valores contenidos en la misma. Pueden llegar a ser de aplicación directa en el derecho, sin necesidad de pasar previamente por las demás formas normativas.

Esta importancia de la Constitución como máximo referente normativo, ha sido definida por García (2010) al señalar que una de las características fundamentales del neoconstitucionalismo se encontraba en el hecho de definir a la Constitución a manera de regente jurídico de mayor alcance y predominancia en la organización estatal, transformándola en garante legal y herramienta judicial de aplicación directa; cuyo papel predominante conferido a la Constitución es precisamente el que asume el Estado de derecho dentro del Estado social y democrático.

Por tanto, la interpretación del derecho basada sólo en la aplicación de las reglas, dentro del ámbito del neoconstitucionalismo implica un nivel interpretativo más amplio, debido a que los principios y derechos constitucionales fundamentales se deben interpretar mediante la ponderación, y de la misma forma, se debe resolver los conflictos que se presenten entre ellos (García, 2010). Al respecto, Bernal (citado por Torres, 2017), resume el sistema de la ponderación en tres pasos, de la siguiente forma: 
En el primer paso, es preciso definir el grado de la no satisfacción o de afectación de uno de los principios. Luego, en un segundo paso, se define la importancia de la satisfacción del principio que juega en sentido contrario. Finalmente, en un tercer paso, debe definirse si la importancia de la satisfacción del principio contrario justifica la afectación o la no satisfacción del otro (Torres, 2017, p. 266).

En el caso colombiano, la Constitución Política (CP, 1991) creó, para la interpretación de su texto la Corte Constitucional, entidad perteneciente a la Rama Judicial, sin embargo, no es la única que aplica dicha interpretación, que más bien se radica dentro de la Rama Judicial del Poder Público, en mayor o menor medida, debido a que de la manera en cómo está organizado el poder judicial, no sólo en todas las instancias se debe aplicar la Carta Política, sino que todos los jueces pueden actuar como Juez Constitucional por vía de tutela. Este tipo de mecanismos constitucionales hacen que el texto constitucional sea directamente aplicable para cualquier ciudadano por medios como la acción de tutela, de esta forma:

[...] permite proteger derechos innominados en las leyes e incluso apartarse de la ley cuando señala una solución diferente de la que se deriva de una interpretación sistemática de la Constitución. Mediante el control de constitucionalidad que ejerce la Corte se garantiza la supremacía y efectividad de la Constitución, pero particularmente del amplio catálogo de derechos que consagra (García, 2008, p. 299).

\section{Igualmente:}

[...] una particularidad concreta del Estado Social de Derecho que [presentó] [...] el Constituyente de 1991, es que la Constitución, además de ser [sobresaliente] [...] en el ordenamiento jurídico, tiene carácter vinculante o fuerza normativa, lo cual significa que vincula a todo el poder público. Dicho, en otros términos; no es apenas un referente formal ni retórico en el sistema de [principios] [...] formales del derecho, "sino que se trata de una verdadera norma jurídica, de carácter vinculante de la constitución que, para el neoconstitucionalismo [...], [también] regula la organización del poder y las fuentes del derecho y genera de modo directo, derechos y obligaciones inmediatamente exigibles (Sentencia T-267, 2010, num. II, inc. 3.2, párr. 3). 
Al respecto, la Corte Constitucional en Sentencia T-1094 (2008) manifestó:

La fuerza normativa de la Constitución significa entonces que se ha constituido de manera definitiva en sí misma en fuente del derecho y por tanto aplicable directamente por los jueces en el cumplimiento de la misión que les ha sido confiada, pasando de ser norma de aplicación indirecta para ser norma que se aplica junto con la ley o incluso frente a ella (num. II, inc. 3.1, párr. 3).

Considerando lo anterior se puede afirmar que dentro de la labor judicial en Colombia se ha constituido, a partir de la Constitución Política de 1991 y la creación de la controversial —aunque mayoritariamente aceptada - Corte Constitucional, una serie de técnicas interpretativas basadas en la instauración de principios y derechos fundamentales desde el nivel Constitucional, que, a pesar de su rango, no se hacen abstractos, sino que hay posibilidad de aplicarlos directamente. Esta labor interpretativa donde se debe tener en cuenta:

[...] la ponderación para aplicar los derechos fundamentales, la proporcionalidad, y la razonabilidad en la protección de tales derechos, la maximización de los efectos normativos de los derechos fundamentales, la proyección horizontal de los derechos fundamentales [...] o debido a la consideración en casos particulares, [es lo que puede denominarse interpretación a la luz de los postulados neoconstitucionalistas] (Estrada, citado en García, 2008, p. 302).

Toda esta labor interpretativa le corresponde -como se había dichoal Juez, cualquiera que sea su especialidad, con las correspondientes instancias judiciales y la posibilidad de que cualquier caso del territorio colombiano sea revisado de manera oficiosa por el máximo intérprete de la Constitución, la Corte Constitucional.

Sin embargo, dejando el caso colombiano y regresando al tenor general del asunto, la implicación de la adopción del Estado Social de Derecho como parte activa y directa de la labor judicial genera una problemática jurídica con respecto al papel de los jueces, que 
versa sobre su independencia y sus deberes. Dicha problemática ha sido excelentemente resumida por Uprimny (citado por García, 2005), quien la presenta en forma de interrogación:

¿Cómo garantizar que unas personas que no son elegidas de forma democrática (los jueces), y que en muchos casos no deben serlo precisamente para que puedan ser independientes y garantizar los derechos de las personas, sin embargo, decidan los casos de manera objetiva y justa, garantizando lo liberal, lo democrático y lo social de este tipo de Estado? (p. 50).

A juicio del autor, para lograr esta garantía es necesario que los jueces respeten tres obligaciones esenciales: (I) El accionar judicial debe certificar los derechos de las personas mediante decisiones judiciales (sentencias) previsibles, sustentadas en el principio de seguridad jurídica y así la administración de justicia no se convierta en un acto arbitrario y no vulnere los derechos y libertades establecidos en la Constitución. (II) Dado que el juez carece de legitimación democrática, es su obligación acatar y respetar las decisiones mayoritarias de los órganos políticos que han sido elegidos por sufragio popular. (III) Los fallos de los jueces deben ser materialmente justas, ya que, en virtud de los principios sociales del Estado social de derecho, la actividad judicial (como todas las otras funciones) debe contribuir a alcanzar una sociedad realmente más justa.

Volviendo al caso colombiano, el respeto a la seguridad jurídica, a los derechos fundamentales, a los principios democráticos, a la voluntad popular, y la necesidad de la búsqueda de la justicia material, como partes indispensables de la administración de la misma en todos sus niveles; han formado parte constante del discurso de la Corte Constitucional, en su extensa jurisprudencia en materia de derechos esenciales, considerada por algunos autores, la más completa que sobre el tema existe en América (Carbonell, 2013). Lo que nos conduce a vislumbrar que los requisitos mencionados por Uprimny (García, 2005), participa al menos de forma tácita, del accionar de la Corte Constitucional colombiana, y por consiguiente permean toda la actividad de la dirección de justicia. 
Habiendo analizado los postulados conceptuales del neoconstitucionalismo, es posible decir que tanto; el estado social de derecho, como la justicia constitucional colombiana, cuentan con una profunda y arraigada influencia neoconstitucional, señalado por múltiples tratadistas, entre otros Gil (2005) y Carbonell (2018). Esta afirmación puede hacerse a partir de dos pilares principales de sustento: el texto mismo de la Constitución Política y el accionar de la Corte Constitucional colombiana.

Respecto de la Constitución, al revisar el texto de la Carta Magna colombiana, tendiendo para ello presentes los seis postulados de Prieto, mencionados con anterioridad como características del neoconstitucionalismo. Vemos que la Constitución colombiana cumple con la totalidad de los mismos: posee fuerza vinculante, es considerada normatividad superior, puede ser aplicada de forma directa mediante sus herramientas y acciones constitucionales, ofrece garantía judicial y control sobre la producción legislativa, tiene un extenso contenido normativo en su amplio articulado, y está investida de un carácter rígido cuyo compendio solo pueda ser cambiado por procedimientos complejos y formales.

Así mismo, ese carácter vinculante de la Constitución:

[...] significa que vincula a todo el poder público. [...] No es apenas un referente formal o retórico en el sistema de fuentes formales del derecho; sino que, se trata de una verdadera norma jurídica [...] que, además, de regular la organización del poder y las fuentes del derecho, genera de modo directo derechos y obligaciones inmediatamente exigibles [a los ciudadanos] (Sentencia T-267, 2010, num. II, inc. 3, párr. 3).

Además, regresando a los preceptos de Carbonell en cuanto a la actividad de los jueces dentro de los sistemas neoconstitucionales, vemos igualmente que la Constitución Política creó, tal como es propio del neoconstitucionalismo, una jurisdicción constitucional especial, representada en la Corte Constitucional colombiana. Asimismo, el accionar de dicha Corte, se ha revestido de un discurso constante sobre la forma en que los derechos y libertades constitucionales, deben 
influenciar y sostener, no sólo, a la administración de justicia, sino, a la totalidad de la acción estatal; y, por lo tanto, vincula a todos los órganos y entidades que conforman el Estado.

Siguiendo con el mismo autor, en el accionar de la Corte Constitucional a la hora de tomar decisiones, vemos que dicho cuerpo colegiado recurre con regularidad a la utilización de herramientas surgidas de la teoría, como "la teoría de los principios y de la técnica de la ponderación" de Alexy (citado por Carbonell, 2013); lo que vendría a ilustrar con claridad, la forma en que tal tribunal constitucional acude sin demasiados miramientos o reservas al uso de contenidos propios del neoconstitucionalismo, al dictar sentencia.

Finalmente, y en cuanto a lo mencionado por Uprimny (García, 2005); respecto de las obligaciones esenciales de los jueces, dentro del estado social de derecho y tal como ya se mencionó; la seguridad jurídica a los derechos fundamentales, a los principios democráticos, a la voluntad popular, y la necesidad de la búsqueda de la justicia material, es parte constante del discurso jurídico de las altas cortes colombianas.

\section{Conclusiones}

Todo lo anterior, lleva a concluir que no sólo la administración de justicia en Colombia y el Estado Social de Derecho colombiano están profundamente influenciados por el neoconstitucionalismo, sino que, además, se convierten en uno de los principales referentes del mismo dentro del continente americano.

El positivismo teórico, basado en principios de legalidad; y donde, el método de interpretación principal es la subsunción, ha ido perdiendo su influencia por el neconstitucionalismo como un fenómeno reciente dentro del Estado Constitucional contemporáneo; que presenta una nueva actitud interpretativa del derecho fundada en las ideas de supremacía del texto constitucional y garantía jurisdiccional.

Fruto del neoconstitucionalismo la interpretación del derecho ha cambiado, pues al colocar la Constitución como referente normativo, cuyo contenido esta plagado de principios y valores hacen que pueda aplicarse de forma directa por los jueces al momento de resolver un 
problema jurídico contenido en un caso difícil por encima de la ley pilar del positivismo jurídico.

Cabe resaltar que la interpretación del derecho implica un ejercicio más complejo, debido a la aplicación de los principios y derechos constitucionales (fundamentales) al caso, predominado la técnica de la ponderación; Resaltando que cuando existan conflictos entre principios se deben aplicar los pasos de la ponderación descritos por Torres (2017).

Fruto de este neoconstitucionalismo la Corte Constitucional colombiana a través del fenómeno jurídico del trasplante complejo utiliza el método de la ponderación para dar solución a los llamados casos difíciles.

Es importante resaltar, que la influencia neoconstitucionalista no solo se observa en la aplicación directa de la constitución radicada principalmente en los funcionarios-jueces de la Rama Judicial, pues dicha influencia irradia a las otras ramas del poder público como la ejecutiva, pues el servidor público que hace parte de la rama ejecutiva al momento de adoptar las decisiones (actos administrativos) de su competencia, deberán tener en cuenta las Sentencias de Unificación de la Corte Constitucional colombiana, guardián de la supremacía e integridad del texto constitucional.

La Constitución, se ha constituido en fuente directa del derecho, dado su carácter vinculante o fuerza normativa que vincula a todo el poder público, por tal razón debe ser aplicada por los jueces sin importar su especialidad o grado, en cumplimiento de su misión, con la opción de ser revisada dicha actuación de manera oficiosa por la Corte Constitucional.

Los postulados del neoconstitucionalismo generan que los jueces desplieguen toda una actividad judicial que comporta un alto grado de "activismo judicial" al momento de resolver los casos difíciles objeto de análisis judicial, toda vez que los ordenamiento positivos vienen sufriendo una serie de cambios al punto de que hoy se mencione la expresión denominada "la constitucionalización del ordenamiento jurídico", al estar impregnado por normas constitucionales que contienen una serie de principios y valores, siendo el principio de la dignidad humana el que irradia todo el texto constitucional. 
Finalmente, hoy en día se cuentan con diversas herramientas de protección de los derechos fundamentales dado el auge del neoconstitucionalismo en la medida en que supone un mejoramiento de los aspectos clásicos del constitucionalismo.

\section{REFERENCIAS}

Barak, A. (2006). The Judge in a Democracy. Princeton: Princeton University Press. Retrieved from https://press.princeton. edu/books/paperback/9780691136158/the-judge-in-a-democracy

Carbonell, M. (2018). ¿Qué es el neoconstitucionalismo? [video de youtube]. Retrieved from https://www.youtube.com/ watch?v=16XmEfzaFGI

Carbonell, M. (2013). Derechos fundamentales y democracia. México, D.F.: Instituto Federal Electoral.

Carbonell, M. (ed.) (2006). Teoría del neoconstitucionalismo. Ensayos escogidos. Madrid: Trotta.

Carbonell, M. y García, L. (2010). El canon neoconstitucional. Bogotá, D.C.: Universidad Externado. Disponible en https:// ideas.repec.org/b/ext/derech/332.html

Comanducci, P. (2002). Formas de (neo)constitucionalismo: un análisis meta-teórico. Isonomía, (16), 89-112. Disponible en http://www.cervantesvirtual.com/obra/formas-de-neoconstitucionalismo--un-anlisis-metaterico-0/

García, L. (2010). Constitución como provisión e irradiación Constitucional. Sobre el concepto de "neoconstitucionalismo" Segunda parte. Estudios de Derecho, 148(66), 91-119. Disponible en https://revistas.udea.edu.co/index.php/red/article/ view/5208

García, J. (2009). Sobre el Neoconstitucionalismo y sus precursores. F. Mantilla (coord.), Controversias constitucionales (pp. 24-69). Bogotá D.C.: UR. Disponible en http://repository. urosario.edu.co/handle/10336/11088 
García, L. (2008). El "nuevo derecho" en Colombia: ¿entelequia innecesaria o novedad pertinente? Revista de derecho, (29), 289-330. Disponible en https://rcientificas.uninorte.edu.co/ index.php/derecho/article/view/2698

García, L. (2005). La renovación de las virtudes del juez en el nuevo derecho sin lagunas: Retos de la labor judicial a partir de la Constitución de 1991. Jurídicas, 2(2), 41-61. Disponible en http://juridicas.ucaldas.edu.co/

Gil, R. (2011). El Neoconstitucionalismo y los derechos fundamentales. Quíd lurís, 12(6), 43-61. Recuperado de https://revistas-colaboracion.juridicas.unam.mx/index.php/quid-iuris/ article/view/17373/15582

Guastini, R. (2007). Estudios de teoría constitucional. (3 ed.). México, D.F.: Fontamara.

León, J. (2015). Consideraciones sobre los procesos de enseñanzaaprendizaje en matería jurídica. En, Ó. Agudelo, M. Prieto \& J. León, Teoría jurídica y enseñanza del derecho (pp. 11-29). Bogotá, D.C.: Universidad Católica de Colombia. Disponible en https://repository.ucatolica.edu.co/handle/10983/18964

Marín, J. y Zuluaga, D. (2013). Estado constitucional: marco institucional esencial para la comprensión de los límites de la reforma constitucional. Criterio Jurídico, 13(1), 141-169. Disponible en https://revistas.javerianacali.edu.co/index.php/ criteriojuridico/article/view/968

Mattos, A. (2001). Implicaciones de la constitucionalización del derecho penal en la dogmatica penal colombiana. Aplicable al tipo penal de falsedad en documento público. Jurídica $M a-$ rio Alario D'Filippo. Cartagena (Colombia), X(19), 48-66. Recuperado de https://repositorio.unicartagena.edu.co/bitstream/handle/11227/7653/2143-4617-1-SM.pdf;jsessionid=1 5FD290F03DD63C183495B4EFAB18EFB?sequence=1

Prieto, L. (2003). Justicia Constitucional y Derechos Fundamentales. Madrid: Trotta. 
Prieto, L. (2001). Neoconstitucionalismo y Ponderación Judicial. Anuario de la Facultad de Derecho de la Universidad Autónoma de Madrid, (5), 201-227. Recuperado de http://afduam.es/wpcontent/uploads/pdf/5/6900111(201-228).pdf

República de Colombia. Asamblea Constituyente. (1991). Constitución Política de la República de Colombia. [Const.]. Gaceta Constitucional $\mathrm{N}^{\mathrm{o}}$ 116. Disponible en http://bit.ly/2NA2BRg

República de Colombia. Corte Constitucional. (19 de abril de 2010). Exps. T-2442387 y T-2482388 acums. [Sentencia T-267]. MP: Juan Carlos Henao Pérez. Disponible en https://www.corteconstitucional.gov.co/relatoria/2010/T-267-10.htm

República de Colombia. Corte Constitucional. (6 de noviembre de 2008). Exp. T-1966364. [Sentencia T-1094]. MP: Clara Inés Vargas Hernández. Disponible en https://www.corteconstitucional. gov.co/relatoria/2008/t-1094-08.htm

Saavedra, M. (1994). Interpretación del derecho y crítica jurídica. México, D.F.: Fontamara.

Torres, H. (2017). El principio de igualdad en la configuración de los regimenes pensionales. Bogotá, D.C.: Universidad Externado. Disponible en https://bdigital.uexternado.edu.co/handle/001/657

Vásquez, G. y Barrios, A. (2018). Supremacía constitucional: enfoque teórico del conflicto de jerarquía, jurisdicción y competencia. Universidad y Sociedad, 10(1), 156-163. Disponible en https:// rus.ucf.edu.cu/index.php/rus/article/view/775

* Está vinculado al proyecto de investigación denominado: "Las Directivas Anticipas" de la maestría en Derecho Constitucional, del grupo de investigación Prospectiva y desarrollo Humano de la Universidad Libre (Cúcuta).

Yamal Elías Leal Esper es Abogado con Especialización de la Universidad Católica (Colombia). Magister en Gestión de la Calidad de la Educación Superior de la Universidad de Pamplona (España). Docenteinvestigador del grupo de investigación Prospectiva y desarrollo humano de la Universidad Libre (Colombia). https://orcid.org/0000-0002-44935866. https://scholar.google.com/citations?user=c5rEajYAAAAJ\&hl=es 